\title{
Role of Limbic System in the Control of Hamster Growth ${ }^{1,2}$
}

\author{
KATARINA TOMLJENOVIĆ BORER, MICHAEL E. TRULSON AND LAWRENCE R. KUHNS \\ Department of Physical Education, The University of Michigan, Ann Arbor MI 48109 \\ Department of Psychology, Princeton University, Princeton NJ 08540 \\ and \\ Department of Radiology, The University of Michigan, Ann Arbor MI 48109
}

(Received 30 October 1978)

\begin{abstract}
BORER, K. T., M. E. TRULSON AND L. R. KUHNS. Role of limbic system in the control of hamster growth. BRAIN RES. BULL. 4(2) 239-247, 1979.--Rostral septal lesions accelerate somatic growth in adult hamsters. This study tested the hypothesis that this effect results from damage to fibers of passage by observing the effects of transections of septohippocampal and septohypothalamic connections on growth. We attempted to identify these fibers further by (a) measuring spectrofluorometrically changes in the monoamine concentrations in hippocampus, cerebral cortex, corpus striatum, and diencephalon, (b) staining the degenerating axons after septal lesions and the two cuts, and (c) examining the correspondence between such damage and the acceleration of growth. Both knife cuts accelerated somatic growth and were associated (as well as septal lesions) with significant depletions of serotonin $(-27$ to $-57 \%)$ and norepinephrine $(-27$ to $-60 \%)$ in the hippocampus, with less consistent depletions of these monoamines in the cerebral cortex, and with no changes in regional dopamine content. All three procedures were associated with degeneration in the hippocampal formation and its fiber systems. Thus, fibers interconnecting hippocampus and brainstem, and passing through septum, exert tonic suppression over somatic growth in adult hamsters.
\end{abstract}

Growth Hippocampus Septum Hypothalamus Brainstem Serotonin Norepinephrine
Dopamine

RODENTS retain the capacity for skeletal and ponderal growth throughout most of their adult lifespan $[2,14,15,26]$, but their growth rate declines around the time of puberty [5, $32,43]$. Although the mechanism responsible for this slowdown in growth rate is not well understood, certain experimental manipulations have revealed a latent capacity for rapid growth in adult hamsters and rats. In adult hamsters, exposure to voluntary exercise $[4,9]$ and lesions of rostral medial septal area [7] are associated with acceleration of somatic and skeletal growth, but not with changes in the percentage of body fat $[7,8]$. In rats, intraventricular administration of a serotonin-depleting drug, 5,7-dihydroxytryptamine to young male animals [40], and transection of anterolateral $[30,31,35]$ and lateral $[20,21]$ connections of the medial basal hypothalamus in adult female animals leads to acceleration of somatic and skeletal growth. These knife cuts are effective only after the females have entered the slow phase of growth characteristic of adult animals [21]. These cuts also induce obesity in rats of both sex [19,35], but do not accelerate somatic growth in males [19,39]. Similarly, acceleration of growth rate in hamsters by voluntary exer- cise only occurs in adult animals whose growth rate has undergone a decline [5]. These findings suggest that growth rate in adult rodents declines under the influence of a growth suppressing mechanism which can be blocked by neuroendocrine manipulations.

In the present study we examined the brain structures and connections which inhibit somatic growth in adult hamsters. Using three experimental approaches, we have tested the hypothesis that the growth acceleration seen following electrolytic lesions of the rostral medial septum [7] results from damage to fibers passing through the septal area and have attempted to identify these fibers. First, we have used a retractable-wire knife [41] to transect axons connecting the septum with hypothalamus and midbrain on one hand and with the hippocampus on the other. Secondly, we have investigated whether the growth-inducing lesions and knife cuts damage serotonergic, noradrenergic or dopaminergic fibers innervating the forebrain by measuring changes in the concentrations of these three monoamines in four regions of the forebrain following the lesions and cuts. Thirdly, using Fink-Heimer staining of degenerating axons, we have sought

'This research was supported in part by grants from the National Institute of Mental Health RO3 MH29877 and PCM-78-07626 from the National Science Foundation to K. T. Borer. We thank Sarah S. Winans for help in evaluation of degeneration staining and for valuable comments which helped improve the manuscript, and Elliot $S$. Valenstein for the use of laboratory equipment and facilities.

"Requests for reprints should be sent to: K. T. Borer, Department of Physical Education. The University of Michigan. 401 Washtenaw Avenue, Ann Arbor MI 48109. 
to determine the similarities in the pattern of neural damage resulting from the different growth-inducing neurosurgical procedures. The evidence from the three experimental approaches strongly implicates neural pathways interconnecting or passing through the hippocampus and brainstem via the septal area in tonic suppression of somatic and skeletal growth in adult hamsters.

\section{METHOD}

\section{Animals}

Ninety-three female golden hamsters weighing about 100 $\mathrm{g}$ and over 100 days old were obtained from Engle Laboratory Animals, Farmersburg IN. Animals were housed individually in standard suspended wire cages in light- (12L:12D) and temperature-controlled $\left(22^{\circ} \mathrm{C}\right)$ rooms with an unlimited supply of Purina Formulab chow pellets and water.

\section{Surgery and Histology}

Hamsters were anesthetized with sodium pentobarbital $(75 \mathrm{mg} / \mathrm{kg}$, IP). All stereotaxic surgery was performed with the skull horizontal between bregma and lambda. For midline septal lesions the stereotaxic coordinates were $2 \mathrm{~mm}$ anterior to bregma, at the midline, and $5 \mathrm{~mm}$ below dura. Two mA of current were passed for $20 \mathrm{sec}$ between the bared tip of an insulated No. 1 insect pin serving as anode and a rectal cathode with a constant-current lesion maker in 10 hamsters. The electrode was lowered $2 \mathrm{~mm}$ below dura but no current was passed in 8 control hamsters. A retractable wire knife [41], $0.15 \mathrm{~mm}$ in diameter, was used for knife cuts. Bilateral hippocampal transections (HIPPO cuts) were done in 22 hamsters. Stereotaxic coordinates were $2.8 \mathrm{~mm}$ posterior to bregma, $0.5 \mathrm{~mm}$ lateral to sagittal sinus, and 3.0 $\mathrm{mm}$ below dura. The knife was directed 45 degrees anterolaterally and adjusted to a length of $3.4 \mathrm{~mm}$ with a $1 \mathrm{~mm}$ vertical dip. The knife was lowered, extended, and raised to the surface of the brain. In 20 control hamsters the same procedure was followed except that the knife was lowered $0.5 \mathrm{~mm}$ below dura. Bilateral septohypothalamic transections ( $\mathrm{SH}$ cuts) were done in 19 hamsters. The stereotaxic coordinates for this cut were $2 \mathrm{~mm}$ anterior to bregma, 1.6 $\mathrm{mm}$ lateral to sagittal sinus and $4.5 \mathrm{~mm}$ below dura. In this position, the knife was extended $2.5 \mathrm{~mm}$ in the anterior direction, rotated medially $180^{\circ}$, retracted and removed. In 14 control hamsters the same procedure was followed except that the retracted knife was lowered $2 \mathrm{~mm}$ below dura and removed unextended.

For monoamine measurements, hamsters were decapitated and their brains were quickly removed and placed on a metal surface in contact with ice. The olfactory lobes were sectioned at the level of frontal poles and discarded. After a sagittal callosal separation, an incision was made along the cingulate gyrus and the cerebral hemispheres were retracted laterally to exposure the ventricle, hippocampus, septum, and corpus striatum. The hippocampi (including the subiculum, hippocampus proper, and dentate gyrus) were peeled free. The corpus striatum (caudate-putamen and globus pallidus) were extracted with fine forceps. The remaining portion of telencephalon including amygdala, entorhinal area, presubiculum and cerebral cortex but excluding the part of the hemisphere lateral to corpus striatum, was separated from the diencephalon with scissors and will be referred to as cerebral cortex. The diencephalon was dissected with a razor blade. Vertical cuts were made caudal to optic chiasm, caudal to mammillary bodies, and medial to optic tracts. The tissue samples were wrapped in aluminum foil, frozen within 5-12 minutes in liquid nitrogen and stored at $-70^{\circ} \mathrm{C}$ for subsequent monoamine determinations.

Ten hamsters were used to study the pattern of neuronal degeneration produced by lesions or knife cuts. The tissue was impregnated with silver according to procedure II of Fink and Heimer [18]. Three-day survival time was used for four animals with midline septal lesions, 4 hamsters with unilateral SH cuts and 2 animals with unilateral HIPPO cuts. Following the intracardiac perfusion with isotonic saline followed by $10 \%$ formol-saline, brains were dissected and allowed to harden in $10 \%$ formol-saline for a week. Brains were then embedded in two parts of egg-yolk mixed with one part of $12 \%$ gelatin ([16] and sectioned at $25 \mu$ on a freezing microtome. Brain sections were stored refrigerated in $1 \%$ Formalin and stained for neuronal degeneration. Adjacent sections were stained with cresyl violet to determine the location of damage and of degenerating pathways.

Concentrations of serotonin, norepinephrine, and dopamine were determined in hippocampus, cerebral cortex, corpus striatum, and diencephalon with a spectrofluorometric method [23]. All of the measurements for a given neurosurgical procedure were made within the same assay. Assays for the three neurosurgical procedures were performed at different times. Recoveries of compounds added to the homogenate were between 84 and $93 \%$ for serotonin, between 94 and $99 \%$ for norepinephrine, and between 80 and $94 \%$ for dopamine. Results have not been corrected for recovery.

\section{Experimental Design and General Procedure}

Animals were matched by body weight during the week preceding surgery and assigned to treatment or control groups. Weight measurements were taken daily. The rates of weight gain (ponderal growth rates) were determined as the slopes of least squares linear regression of body weight as a function of time after surgery. Animals which were used for the determinations of brain monoamine concentrations were killed between the eighth and twelfth postoperative days during the period of maximal acceleration of ponderal growth. Skeletal growth was evaluated from the whole-body radiographs on the 55th postoperative day in 10 hamsters with HIPPO cuts and in 10 control-operated animals, and on the 77 th postoperative day in 7 hamsters with SH cuts and in 6 surgical controls according to a standardized procedure [9]. Length measurements of the skull, the vertebral column of the body and the tail, of left humerus and the left femur were taken with direct-reading point calipers from the whole-body radiographs of experimental and control hamsters. Hamsters were anesthetized and extended on a Plexiglas device which insured constant body position. Radiographs were taken on Dupont Cronex film with a portable General Electric X-ray machine at a constant distance of $107 \mathrm{~cm}$ between the $X$-ray tube and the film and with exposures of $50 \mathrm{KV}, 80$ milliamperessecond [9].

\section{Data Analysis}

Standard errors of the mean are used to express data variability. Results were evaluated with the Student's $t$-test, two-tailed except for skeletal growth data for which a onetailed test was used. 


\section{RESULTS}

Acceleration of Growth by Midline Septal Lesions and by Bilateral HIPPO and SH Cuts

The effectiveness of midline electrolytic lesions of rostral medial septal area in accelerating ponderal and skeletal growth in adult hamsters was recently described [7]. We have replicated this phenomenon in the present study with 6 lesioned hamsters whose postoperative weight changes are presented in Fig. 1, left. Following a 9-g weight loss on the first postoperative day, hamsters with septal lesions gained $3.1 \pm 0.4 \mathrm{~g} /$ day compared to ponderal growth of $0.3 \pm 0.1$ $\mathrm{g} /$ day in 8 control animals $(p<0.001)$. Ten hamsters with HIPPO cuts lost $10 \mathrm{~g}$ on the first postoperative day and subsequently gained $1.8 \pm 0.2 \mathrm{~g} /$ day compared to the rate of weight gain in 10 control hamsters of $0.3 \pm 0.1 \mathrm{~g} /$ day (Fig. 1, middle, $p<0.001)$. Increased ponderal growth rate $(2.0 \pm 0.1$ vs. $0.2 \pm 0.1 \mathrm{~g} /$ day in control animals $p<0.001)$ continued during the first 30 postoperative days in 10 hamsters with HIPPO cuts that were allowed extended postoperative survival. On Day 55 after surgery all skeletal measurements of the HIPPO-cut animals were significantly greater than in 10 control hamsters (Table 1).

SH cuts in 8 hamsters were associated with a $20 \mathrm{~g}$ weight loss during the first two postoperative days followed by an acceleration of ponderal growth rate to $2.5 \pm 0.3 \mathrm{~g} / \mathrm{day}$ compared to the control rate of $0.0 \pm 0.1 \mathrm{~g} /$ day in 8 animals (Fig. 1 , right, $p<0.001$ ). Accelerated ponderal growth was also observed during 30 days following surgery $(2.0 \pm 0.1$ vs. $0.4 \pm 0.1 \mathrm{~g} /$ day in controls, $p<0.001)$ in 7 hamsters with $\mathrm{SH}$ cuts allowed extended postoperative survival. On the 77 th postoperative day all but one of the skeletal length measurements in SH cut animals were significantly greater than in 6 control animals (Table 1).

Changes in Regional Brain Monoamine Concentrations Following Medial Septal Lesions and Bilateral HIPPO and SH Knife Cuts

Significant depletions of serotonin (Table 2) and norepinephrine (Table 3 ) in the hippocampus were noted after all three neurosurgical procedures. Serotonin depletions in hippocampus ranged between 27 and 57\%, while norepinephrine depletions in hippocampus ranged between 27 and $60 \%$. In each case, depletions of monoamines were lowest following septal lesions and greatest after SH knife

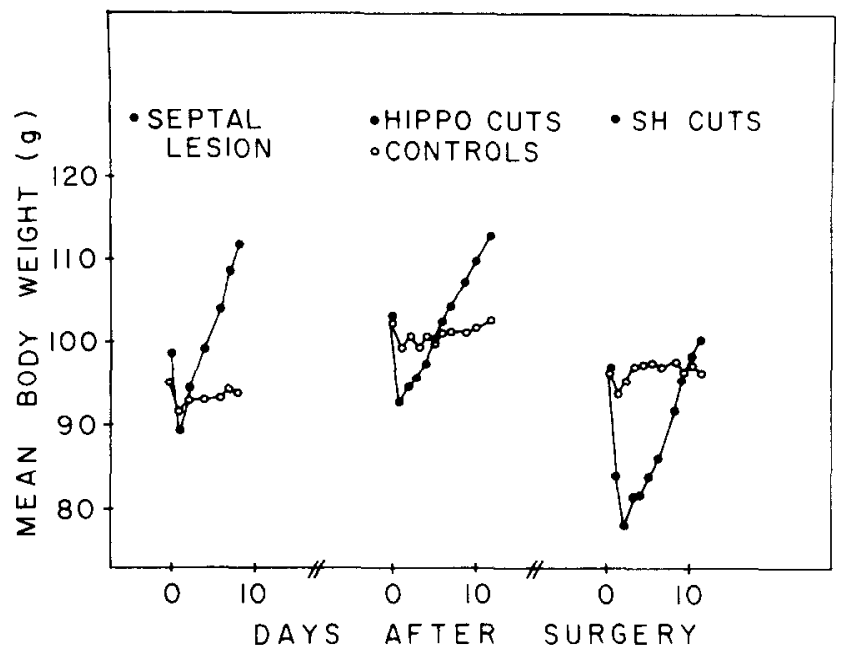

FIG. 1. Mean weight changes of adult hamsters receiving midline septal lesion $(n=6)$, bilateral transection of dorsal hippocampus (HIPPO cuts, $n=10$ ), or bilateral horizontal cuts separating septum and hypothalamus (SH cuts, $\mathrm{n}=8$ ) and of control-operated hamsters $(n=8, n=10, n=8$, respectively).

cuts. In addition there was a significant depletion of serotonin in the cerebral cortex, ranging between 9 and 22\%, after all three neurosurgical procedures (Table 2) and significant depletion of cortical norepinephrine after HIPPO $(-17 \%)$ and SH (-30\%) knife cuts (Table 3). With one exception (23\% increase in diencephalon after HIPPO cuts), there was no significant change in regional concentrations of dopamine after either of the three neurosurgical procedures (Table 4).

\section{Location of Brain Damage after Midline Septal Lesions and Bilateral HIPPO and SH Cuts}

Location and size of brain damage in the septal-lesioned animal showing greatest acceleration of growth $(4.0 \mathrm{~g} / \mathrm{day}, 6)$ is shown in Fig. 2. The center of the lesion was located in the midline 0.5 to $1.0 \mathrm{~mm}$ anterior to the anterior commissure. Damage was seen in medial septal nucleus, medial part of lateral septal nucleus, vertical limb of the diagonal band of

TABLE 1

INCREASED SKELETAL GROWTH IN ADULT HAMSTERS WITH HIPPOCAMPAL TRANSECTIONS AND SEPTOHYPOTHALAMIC CUTS

\begin{tabular}{lccccc}
\hline & \multicolumn{5}{c}{ Lengths (mm) } \\
& Skull & Body & Tail & Humerus & Femur \\
\hline Postoperative Day 55 & & & & & \\
$\quad$ HIPPO cut $(n=10)$ & $37.21 \pm 0.15^{*}$ & $112.75 \pm 0.73^{*}$ & $35.41 \pm 0.35 \dagger$ & $25.06 \pm 0.17^{*}$ & $29.43 \pm 0.12^{*}$ \\
Control $(\mathrm{n}=10)$ & $36.40 \pm 0.13$ & $105.41 \pm 0.61$ & $33.53 \pm 0.39$ & $23.92 \pm 0.15$ & $27.87 \pm 0.11$ \\
Postoperative Day 77 & & & & & \\
SH cut $(\mathrm{n}=7)$ & $38.02 \pm 0.21^{\dagger}$ & $118.31 \pm 0.46^{*}$ & $35.43 \pm 1.57$ & $25.89 \pm 0.08^{*}$ & $29.26 \pm 0.27^{*}$ \\
Control $(\mathrm{n}=6)$ & $36.71 \pm 0.27$ & $107.20 \pm 1.22$ & $33.00 \pm 1.00$ & $24.36 \pm 0.16$ & $27.27 \pm 0.29$ \\
\hline
\end{tabular}

*Significantly different from corresponding control group: $(p<0.0005)$

$\uparrow$ Significantly different from corresponding control group: $(p<0.005)$. 
TABLE 2

REGIONAL SEROTONIN CONCENTRATIONS $(\mu G / G)$ FOLLOWING MIDLINE SEPTAL LESION AND BILATERAL HIPPO AND SH KNIFE CUTS

\begin{tabular}{|c|c|c|c|c|c|}
\hline Regions & $N$ & Hippocampus $\%$ & $\begin{array}{l}\text { Cerebral } \\
\text { Cortex } \%\end{array}$ & $\begin{array}{c}\text { Corpus } \\
\text { Striatum } \%^{*}\end{array}$ & Diencephalon $\%^{*}$ \\
\hline \multicolumn{6}{|l|}{ Surgery } \\
\hline Septals & 6 & $1.021 \pm 0.05-27.08$ & $0.610 \pm 0.01-9.4 \div$ & $1.632 \pm 0.07+0.9$ & $1.653 \pm 0.03+2.9$ \\
\hline Controls & 8 & $1.399 \pm 0.04$ & $0.673 \pm 0.01$ & $1.618 \pm 0.06$ & $1.607 \pm 0.03$ \\
\hline HIPPO cuts & 10 & $0.308 \pm 0.02-50.18$ & $0.359 \pm 0.01-12.4 t$ & $0.610 \pm 0.02+6.3$ & $0.925 \pm 0.02+4.6$ \\
\hline Controls & 10 & $0.617 \pm 0.02$ & $0.410 \pm 0.02$ & $0.574 \pm 0.03$ & $0.884 \pm 0.03$ \\
\hline SH cuts & 8 & $0.311 \pm 0.03-56.78$ & $0.368 \pm 0.017-21.7 \frac{7}{2}$ & $0.592 \pm 0.03-2.0$ & $1.050 \pm 0.03+0.6$ \\
\hline Controls & 8 & $0.719 \pm 0.02$ & $0.470 \pm 0.022$ & $0.604 \pm 0.03$ & $1.044 \pm 0.05$ \\
\hline
\end{tabular}

* Percent change relative to the corresponding control group.

t Significantly different from the corresponding control group: $(p<0.02)$.

$\mp$ Significantly different from the corresponding control group: $(p<0.01)$

$\$$ Significantly different from the corresponding control group: $(p<0.001)$.

TABLE 3

REGIONAL NOREPINEPHRINE CONCENTRATIONS $(\mu \mathrm{G} / \mathrm{G})$ FOLLOWING MIDLINE SEPTAL LESION AND BILATERAL HIPPO AND SH KNIFE CUTS

\begin{tabular}{lrllll}
\hline Regions & $\mathrm{N}$ & \multicolumn{1}{c}{ Hippocampus $\%^{*}$} & \multicolumn{1}{c}{ Cortex $\%^{*}$} & Striatum \%* & Diencephalon $\%^{*}$ \\
\hline Surgery & & & & & \\
$\quad$ Septals & 6 & $0.332 \pm 0.05-26.9 \pm$ & $0.377 \pm 0.04-8.3$ & $0.409 \pm 0.05$ & $1.713 \pm 0.14+9.7$ \\
Controls & 8 & $0.454 \pm 0.02$ & $0.411 \pm 0.02$ & $0.409 \pm 0.03$ & $1.561 \pm 0.06$ \\
HIPPO cuts & 10 & $0.175 \pm 0.01-37.9 \$$ & $0.226 \pm 0.01-16.6 \pm$ & $0.422 \pm 0.02+3.9$ & $1.331 \pm 0.04+6.0$ \\
Controls & 10 & $0.282 \pm 0.02$ & $0.271 \pm 0.01$ & $0.406 \pm 0.04$ & $1.256 \pm 0.04$ \\
SH cuts & 8 & $0.128 \pm 0.01 \cdots 60.4 \$$ & $0.166 \pm 0.02-30.3$ & $0.302 \pm 0.01-14.4$ & $1.172 \pm 0.03+5.5$ \\
Controls & 8 & $0.323 \pm 0.01$ & $0.238 \pm 0.01$ & $0.353 \pm 0.02$ & $1.111 \pm 0.06$ \\
\hline
\end{tabular}

*Percent change relative to the corresponding control group.

tSignificantly different from the corresponding control group: $(p<0.05)$.

$\$$ Significantly different from the corresponding control group: $(p<0.01)$

$\$$ Significantly different from the corresponding control group: $(p<0.001)$.

TABLE 4

REGIONAL DOPAMINE CONCENTRATIONS $(\mu \mathrm{G} / \mathrm{G})$ FOLLOWING MIDLINE SEPTAL LESION AND BILATERAL HIPPO AND SH KNIFE CUTS

\begin{tabular}{lrllll}
\hline Region & $\mathrm{N}$ & \multicolumn{5}{c}{ Hippocampus $\%^{*}$} & \multicolumn{1}{c}{$\begin{array}{c}\text { Cerebral } \\
\text { Cortex } \%^{*}\end{array}$} & $\begin{array}{c}\text { Corpus } \\
\text { Striatum } \%^{*}\end{array}$ & Diencephalon \%* \\
\hline Surgery & & & & & \\
$\quad$ Septals & 6 & $0.795 \pm 0.05-3.0$ & $0.388 \pm 0.05-2.8$ & $6.557 \pm 0.68-15.5$ & $1.029 \pm 0.15-22.1$ \\
Controls & 8 & $0.820 \pm 0.05$ & $0.399 \pm 0.03$ & $7.757 \pm 0.24$ & $1.321 \pm 0.03$ \\
HIPPO cuts & 10 & $0.689 \pm 0.07+8.5$ & $0.372 \pm 0.04+7.8$ & $8.494 \pm 0.61+6.1$ & $1.235 \pm 0.08+22.5+$ \\
Controls & 10 & $0.635 \pm 0.12$ & $0.345 \pm 0.04$ & $8.003 \pm 0.87$ & $1.008 \pm 0.07$ \\
SH cuts & 8 & $0.655 \pm 0.07-9.8$ & $0.317 \pm 0.04-6.5$ & $6.686 \pm 0.49-13.2$ & $1.286 \pm 0.09+6.4$ \\
Controls & 8 & $0.726 \pm 0.05$ & $0.339 \pm 0.03$ & $7.710 \pm 0.40$ & $1.209 \pm 0.09$ \\
\hline
\end{tabular}

*Percent change relative to the corresponding control group.

$\uparrow$ Significantly different from the corresponding control group: $(p<0.05)$. 

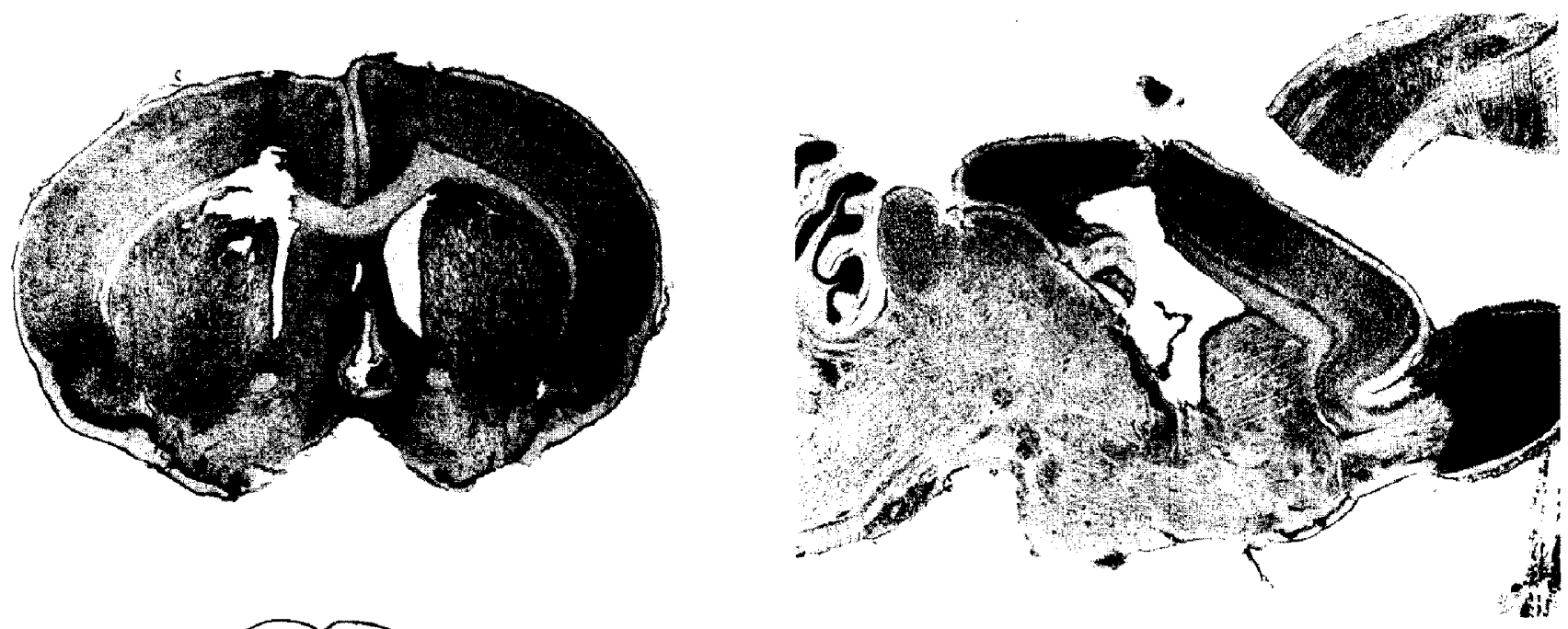
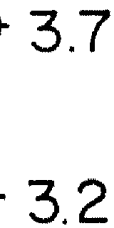

$+2.8$

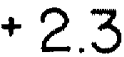

$+1.8$

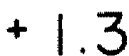

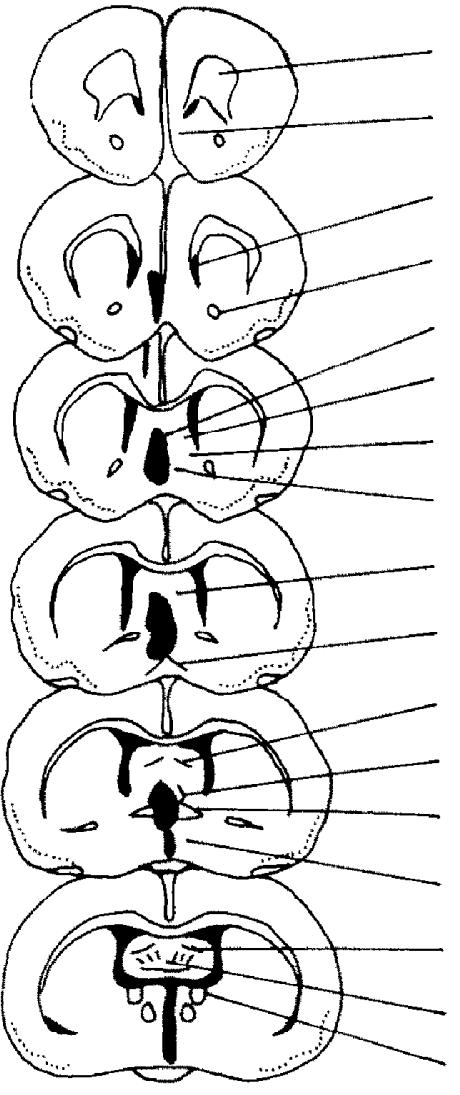

GC C

$\mathrm{sm}$

$V L$ CAA $\mathrm{s} m$ s!

a TD FPC I R LAT FO $F$ CA pom FO ts SM

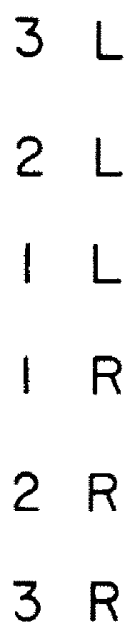

FIG. 2. (A) Coronal view of the center of a midline rostral septal lesion in a hamster showing a postoperative ponderal growth rate of $4.0 \mathrm{~g} / \mathrm{day}$. (B) Reconstruction of location and extent of histological damage in the same hamster. Numbers indicate in $\mathrm{mm}$ distance with respect to bregma. Abbreviations: a, nucleus accumbens, CA, anterior commissure, CAA, anterior limb of the anterior commissure, $\mathrm{CC}$, corpus callosum, $\mathrm{cp}$, caudate putamen, $\mathrm{F}$, column of the fornix, FO, fornix, FPC, precommissural fornix, GCC, genu of corpus callosum, ha, anterior hypothalamic nucleus, HI, hippocampus, hvm, ventromedial hypothalamic nucleus, LAT, lamina terminalis, pom, medial preoptic nucleus, sl, lateral septal nucleus, sm, medial septal nucleus, SM, stria medullaris, TD, tract of the diagonal band of Broca, TO, optic tract, ts, triangular septal nucleus, VL, lateral ventricle, V III, third ventricule.

\section{HIPPO CUT}

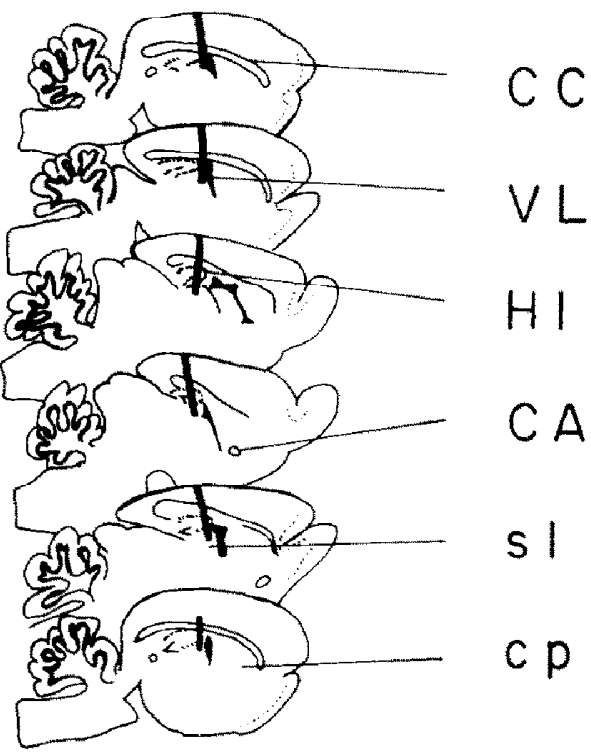

FIG. 3. (A) Sagittal view, about $1 \mathrm{~mm}$ lateral to midline of a transection of dorsal hippocampus in a hamster with the postoperative weight gain of $2.4 \mathrm{~g} /$ day. (B) Reconstruction of location and extent of histological damage in the same hamster. Numbers indicate in $\mathrm{mm}$ distance of the section from midline. Letters $\mathrm{L}$ (left) and $\mathrm{R}$ (right) indicate direction with respect to midline. Abbreviations as in Fig. 2.

Broca, and the fornix at the point of the formation of the descending columns.

The location and extent of brain damage in the HIPPO-cut hamster showing the greatest acceleration of growth $(2.4$ g/day) is presented in Fig. 3. The cut transected the dorsal hippocampus and the overlying corpus callosum and cerebral cortex approximately $1.5 \mathrm{~mm}$ posterior to the hippocampal transition into fornix. In the control hamsters damage was confined to cerebral cortex, corpus callosum, and, in some animals, to the dorsal aspect of the hippocampus. Location and extent of brain damage in the SH-hamster show- 


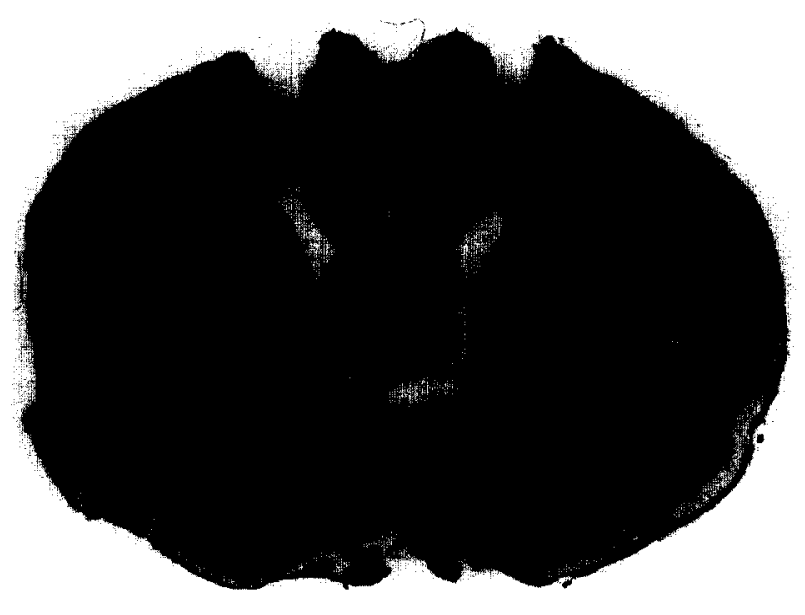

\section{$\mathrm{SH} \quad \mathrm{CUT}$}
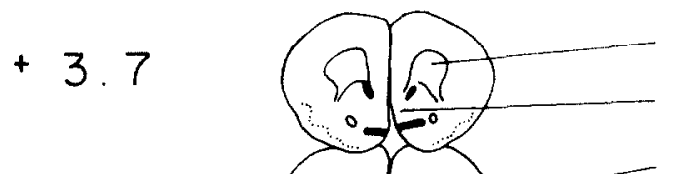

$+3.2$

$+2.8$

$+2.3$

$+1.8$

$+1.3$

$+0.6$

0.0
GCC

$\mathrm{s} \mathrm{m}$

$V L$

$C A A$

$s m$

s I

a

$T D$

FPC

LAT

$\mathrm{F}$

$C A$

pom

$\mathrm{FO}$

tS

$S M$

ha

HI
FO

FIG. 4. (A) Coronal view of the midpoint of a bilateral horizontal cut in a harnster with a postoperative weight gain of $2.5 \mathrm{~g} /$ day. (B) Reconstruction of location and extent of histological damage in another hamster with comparable postoperative weight gain. Abbreviations as in Fig. 2.

ing greatest acceleration of growth $(2.5 \mathrm{~g} / \mathrm{day})$ is shown in Fig. 4. The cut was located at the level of the anterior commissure and extended from the rostral end of the medial septal nucleus to the level of the anterior thalamus. SH cuts transected the vertical limb of the diagonal band of Broca, separated the septum from the preoptic and hypothalamic areas and interrupted the stria medullaris and the descending columns of the fornix. SH cuts were often associated with distension of the third ventricle.

Patterns of Neuronal Degeneration after Septal Lesions and Bilateral HIPPO and SH Cuts

Anterior to the septal lesion degeneration was seen along the midline, curving dorsolaterally toward the cingulum and across the corpus callosum (Fig. 5). Degenerating fibers were seen radiating from the cingulum bundle to the cingulate and prefrontal cortex. Ventral to the lesion degeneration was seen along the midline and travelling ventrolaterally in the vertical limb of the diagonal band of Broca. Posterior to the lesion, degeneration was prominent in the hippocampal complex (fimbria, fornix, hippocampal fields $\mathrm{CA}_{1}, \mathrm{CA}_{2}, \mathrm{CA}_{3}$, dorsal and ventral hippocampal commissures, and the descending columns of the fornix) in the medial corticohypothalamic tract, stria medullaris, external capsule and the medial forebrain bundle.

Anterior to the HIPPO cut degeneration was seen in the rostral medial septal nucleus, cingulum and cingulate cortex, corpus callosum, vertical limb of the diagonal band of Broca, throughout the hippocampal formation, in descending columns of the fornix and the medial corticohypothalamic tract, stria medullaris, external capsule, and the medial forebrain bundle. Posterior to the cut degeneration was seen in the anteromedial thalamic nucleus, ventromedial, arcuate, and premammillary nuclei, and the fasciculus retroflexus.

At the anterior end of the SH cut degeneration was seen above and below the cut: dorsally, in the medial septum, cingulum, cingulate cortex, and corpus callosum; ventrally, in the vertical limb of the diagonal band of Broca and the medial forebrain bundle.

At the rostro-caudal midpoint of the $\mathrm{SH}$ cut, degeneration was more prominent dorsal to the cut (in the fornix at the point of the formation of the descending columns, in the cingulum bundle and in corpus callosum), than ventral to the cut, where some degeneration was noted in the medial forebrain bundle and the pyriform cortex. Degeneration was alse seen in the medial corticohypothalamic tract. More posteriorly, degeneration above the cut involved medial and lateral habenulae, stria medullaris, fimbria, cingulum, anteromedial thalamic nucleus, and corpus callosum. Below the cut, medial forebrain bundle, ventromedial hypothalamic nucleus, ventral tegmental area, and columns of the fornix showed degeneration.

\section{DISCUSSION}

Our objective was to delineate brain pathways which participate in tonic suppression of growth in adult hamsters. Our starting point was the observation that electrolytic destruction of rostral medial septal nucleus in adult hamsters lead to a large acceleration of ponderal and skeletal growth, increased secretion of growth hormone and insulin, and depletion of growth hormone from the anterior pituitary. None of these changes are seen after septal lesions in hypophysectomized hamsters [7] suggesting that such lesions destroy 


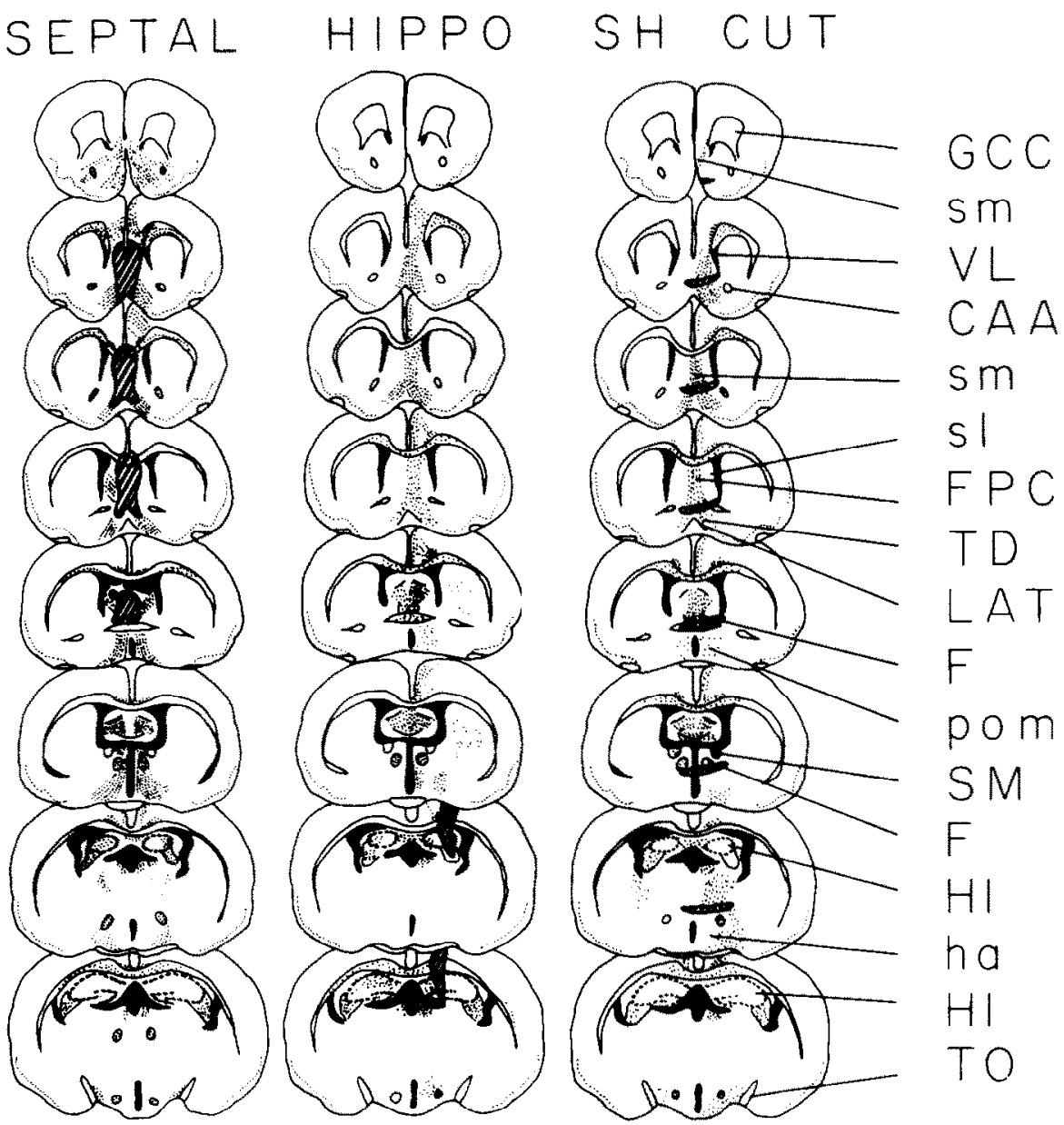

FIG. 5. The pattern of neuronal degeneration revealed by Fink-Heimer staining in representative hamsters with a midline rostral septal lesion (left), unilateral HIPPO cut (middle), and unilateral SH cut (right). Abbreviations as in Fig. 2.

neurons which maintain the slow rate of somatic growth in adult hamsters through their inhibitory influence over growth hormone secretion.

In the present study we have extended the information about the growth-inhibitory neurocircuitry in adult hamsters in three ways. First, we have shown by means of the knifecut analysis, that the growth-inhibitory function is not confined to the neurons in the rostromedial septal area, but is mediated by nerve fibers passing through the septal area. We show that the knife cuts placed in dorsal hippocampus (HIPPO cuts) which transect fibers interconnecting septal area and hippocampal formation $[29,33,34,37,45]$ and cuts placed between septum and hypothalamus ( $\mathrm{SH}$ cuts) are both effective in accelerating ponderal and skeletal growth in adult hamsters. Elsewhere, we also show that HIPPO [6] and $\mathrm{SH}$ cuts [10] are also effective in increasing serum concentrations of growth hormones and insulin. Such evidence suggests, but does not prove, that growth acceleration seen after lesion and the two knife cuts reflects damage to a common growth-inhibiting fiber system which passes through the rostromedial septal area. Somewhat greater acceleration of growth in septal-lesioned hamsters than in HIPPO- and $\mathrm{SH}$-cut animals, revealed by comparison of mean ponderal growth rates (Fig. 1) as well as of maximal growth rates attained by individual animals, suggests that septal area may serve not only as an area of transit for the growth-inhibitory fibers, but as an integrative center as well.

Our second finding was that hippocampal formation serves as origin, destination, or relay for the growthinhibitory fiber system in adult hamsters. Hippocampal involvement in the suppression of growth in adult hamsters was evident from the correspondence between the growth acceleration seen after the three neurosurgical procedures and the anatomical and neurochemical evidence of damage to hippocampal fiber systems. The knife cut analysis showed that transection of dorsal hippocampus, $1.5 \mathrm{~mm}$ posterior to hippocampal transition into fornix and far removed from septal area, resulted in growth acceleration very similar to that seen after septal lesions. Degeneration staining showed evidence of damage to hippocampus and its efferent pathways, the descending columns of the fornix and the medial corticohypothalamic tract, after all three growth-inducing neurosurgical procedures. Regional determinations of monoamine concentrations in hamster brain showed large and consistent depletions of the same two monoamines in the hippocampus after all three neurosurgical procedures. Such 
consistent correspondence between the acceleration of growth and damage to neural fibers to or from the hippocampal formation is taken as evidence that hippocampal fibers or fibers passing through hippocampal formation participate in tonic suppression of growth in adult hamsters.

The third finding of this study implicates two specific monoamine pathways as the possible mediators of growth inhibition in adult hamsters. Neurosurgically-induced acceleration of growth was associated with large and consistent depletions of hippocampal (and to lesser extent cerebral cortical) serotonin (Table 2) and norepinephrine (Table 3) and with no consistent changes in brain dopamine (Table 4). Since it is known that serotonergic fibers from nucleus raphe medianus $[13,27,44]$ and dorsal noradrenergic fibers from locus coeruleus $[1,3,24,36]$ project selectively to hippocampal formation and cerebral cortex, and that depletions of these target tissues after direct lesions to serotonergic [27] and noradrenergic [1] pathways are of the same order of magnitude as those noted in the present study, we conclude that our neurosurgical procedures probably damage these fiber systems as they traverse hypothalamus ( $\mathrm{SH}$ cuts), turn dorsally toward hippocampus (septal lesion) and approach target cells in hippocampus (HIPPO cuts). Direct destruction of noradrenergic neurons in locus coeruleus and of serotonergic neurons in the nucleus raphe medianus will be necessary to determine unequivocally the involvement of these two fiber systems in the control of growth in adult hamsters. In addition, the possible involvement of the cholinergic pathways in the tonic suppression of growth in adult hamsters should also be examined, because of the close proximity of the cholinergic $[25,42\}$ and monoaminergic projections to hippocampus and cerebral cortex.

Evidence generated in this study shows a close correspondence between the acceleration of growth in adult hamsters and damage to nerve fibers interconnecting brain stem and hippocampal formation and passing through the rostromedial septum. This study, however. does not provile clear evidence for the identity of fibers that connect the above neurocircuitry with its endocrine effector, the anterior pituitary gland. We have observed degenerating neurons in the medial corticohypothalamic tract, which, according to some investigators [38] is a hippocampal and limbic neuroendocrine effector pathway. Other possible candidates, suggested by data generated in the rat, are neurons impinging onto the suprachiasmatic nucleus $[46]$, and somatostatinergic neurons interconnecting the periventricular hypothalamic nucleus and the median eminence area $[12,17]$. These pathways have been implicated in the neuroendocrine suppression of growth hormone secretion in the rat, and could, perhaps, participate in the suppression of somatic growth in this species as well as in the hamster. On the other hand, consideration of data obtained in the rat may not be valid in studies of the control of growth in adult hamsters because of the apparently great species differences in the neural circuits controlling growth. In the rat, neither septal lesions $[11,48]$ nor hippocampal damage 147$]$ facilitate growth hormone secretion and somatic growth, and electrical stimulation of hippocampal formation is associated with increased growth hormone secretion [28]. The only similarities between the two species are evidence that serotonin [40] and a longitudinal fiber system interconnecting brainstem and telencephalon [20] may mediate tonic suppression of growth in both. However, large sex differences in the body size, somatic growth $[43]$ and in the effectiveness of telencephalic and mesencephalic $[19,22,39]$ knife cuts in accelerating growth in adult rats, and absence of these obstacles in adult hamsters, make hamster the species of choice in studies analyzing neuroendocrine controls of growth in adult mammals.

\section{REFERENCES}

1. Anden, N. E., A. Dahlstrom, K. Fuxe. K. Larsson, L. Olson and $U$. Ungerstedt. Ascending monoamine neurons to the telencephalon and diencephalon. Acta phwiol. scand . 67:313-326. 1969.

2. Berg, B. N. and C. R. Harmison. Growth, disease, and aging in the rat. J. Gerontol. 12: 370-377, 1952.

3. Bohus, B. The hippocampus and the pituitary-adrenal system hormones. In: The Itippocampus, Volume 1: Strtecture and Development, edited by R. L. Isaacson and K. H. Pribram. New York: Plenum Press, 1975, pp. 323-353.

4. Borer, K. T. Absence of weight regulation in exercising hamsters. Physiol. Behav. 12: 589-597, 1974.

5. Borer, K. T. and L. R. Kaplan. Exercise-induced growth in golden hamsters: Effects of age, weight, and activity level. Physiol. Behav. 18: 29-34, 1977.

6. Borer, K. T., R. P. Kelch, J. Peugh and C. Huseman. Increased serum growth hormone and somatic growth in adult hamsters with hippocampal transections. Neuroendocrinology, 1979, in press.

7. Borer, K. T., R. P. Kelch, M. P. White, L. Dolson and L. R. Kuhns. The role of septal area in the neuroendocrine control of growth in the adult golden hamster. Neuroendocrinology 23: 33-50, 1977

8. Borer, K. T. and A. A. Kooi. Regulatory defense of the exercise-induced weight elevation in hamsters. Beha'. Biol. 13: 301-310, 1975.

9. Borer, K. T. and L. R. Kuhns. Radiographic evidence for acceleration of skeletal growth in adult hamsters by exercise. Grow $h$ 41: 1-13, 1977.
10. Borer, K. T., N. L. Peters, R. P. Kelch, A. N. Tsai and S. Holder. Contribution of growih, fatness, and activity to weight disturbance following septohypothalamic cuts in adult hamsters. J. comp. physiol. Psychol., 1979, in press.

11. Brown, G. M., I. V. Uhlir, T. Seggie, A. V. Schally and A. T. Kastin. Effect of septal lesions on plasma levels of MSH, corticosterone, $\mathrm{GH}$ and prolactin before and after response to novel environment. Role of MSH in the septal syndrome. Endocrinology 94: 583-587, 1974.

12. Brownstein, M. J., A. Arimura, R. Fernandex-Durango, A. V Schally, M. Palkovits and T. S. Kizer. The effect of hypothalamic deafferentation on somatostatin-like activity in the rat brain. Endocrinology 100: 246-249. 1977.

13. Conrad, L. C. A., C. M. Leonard and D. W. Pfaff. Connections of the median and dorsal raphe nuclei in the rat. An autoradiographic and degeneration study. $J$, comp. Nemol. 156: 179-216, 1974.

14. Dawson, A. B. The persistence of the cartilage plates in the long bones of the white rats. Anat. Rec, 27:202 (Abstract), 1925.

15. Donaldson, H. H. and S. B. Conrow, Quantitative studies on the growth of the skeleton of the albino rat. Am. J. Anat. 26: $237-314,1919$

16. Ebbesson, S. O. E. The selective silver-impregnation of degenerating axons and their synaptic endings in non-mammalian species. In: Contemporary Research Methods in Neuroanatomy, edited by W. J. H. Nauta and S. O. E. Ebbesson. New York: Springer, 1979, pp. 132-161. 
17. Elde, R., T. Hokfelt, O. Johansson, S. Efendic and R. Luft. Somatostatin containing pathways in the nervous system. Abstract 1084, Society for Neuroscience Program. Toronto, Canada, 1976 .

18. Fink, R. P. and L. Heimer. Two methods for selective silver impregnation of degenerating axons and their synaptic endings in the central nervous system. Brain Res. 4: 369-374, 1967.

19. Gold, R. M., J. R. Ieni and E. L. Simson. Delayed or precocious hyperphagia after symmetrical or asymmetrical hypothalamic knife cuts in male and female weanling rats, Physiol. Behov. 18: 275-281, 1977.

20. Gold, R. M., A. P. Jones and P. E. Sawchenko. Paraventricular area: Critical focus of a longitudinal neurocircuitry mediating food intake. Physiol. Behav: 18: 1111-1119, 1977

21. Gold, R. M. and G. Kapatos. Delayed hyperphagia and increased body length after hypothalamic knife cuts in weanling rats. J. comp. phusiol. Psuchol. 88: 202-209, 1975.

22. Grossman, S. P. and T. W. Hennessy. Differential effects of cuts through the posterior hypothalamus on food intake and body weight in male and female rats. Physiol. Behav. 17: 89 $102,1976$.

23. Jacobowitz, D. M. and J. S. Richardson. Method for the rapid determination of norepinephrine, dopamine, and serotonin in the same brain region. Pharmac. Biochem. Behat. 8: 515-519, 1978.

24. Koda, L. Y., J. A. Schulman and F. E. Bloom. Ultrastructural identification of noradrenergic terminals in rat hippocampus: Unilateral destruction of the locus coeruleus with 6-hydroxydopamine. Brain Res. 145: 190-195, 1978.

25. Kuhar, M. J. Cholinergic neurons: Septal-hippocampal relations. In: The Hippocampus, Volume l: Structure and Development, edited by R. L. Isaacson and K. H. Pribram. New York: Plenum Press, 1975, pp. 269-283.

26. Lesser, G. T., S. Deutsch and J. Markofsky. The rat fat-free body in middle life: Continuing growth and histochemical changes. J. Gerontol. 25: 108-114, 1970.

27. Lorens, S. A. and H. C. Guldberg. Regional 5-hydroxytryptamine following selective midbrain raphe lesions in the rat. Bruin Res. 78: 45-56, 1974.

28. Martin, J. B., J. Kontor and P. Mead. Plasma GH responses to hypothalamic, hippocampal and amygdaloid electrical stimulation: Effects of variation in stimulus paraneters and treatment with $\alpha$-methyl-p-tyrosine ( $\alpha$-MT) . Endocrinology 92: 1354-1360, 1973.

29. Meibach, R. C. and A. Siegel. Efferent connections of the hippocampal formation in the rat. Brian Res. 124: 197-224, 1977.

30. Mitchell, J. A., R. Smyrl, M. Hutchins, W. J. Schindler and V. Critchlow. Plasma growth hormone levels in rats with increased naso-anal length due to hypothalamic surgery. Neuroendocrinology 10: $31-45,1972$.

31. Mitchell, J. A., M. Hutchins, W. J. Schindler and V. Critchlow. Increase in plasma growth hormone concentration and nasoana length in rats following isolation of medial basal hypothalamus. Neuroendocrinology 12: 161-173, 1973.
32. Monteiro, L. S. and D. S. Falconer. Compensatory growth and sexual maturity in mice. Anim. Product. 8: 179-192, 1966.

33. Moore, R. Y. Monoamine neurons innervating the hippocampal formation and septum: Organization and response to injury. In: The Hippocampus, Volume I: Siructure and Development. edited by R. L. Isaacson and K. H. Pribram. New York: Plenum Press, 1975, pp. 215-232.

34. Nauta, W. J. H. An experimental study of the fornix system in the rat. $J$. comp. Neurol. 104: 247-270, 1956 .

35. Palka, Y., R. A. Liebelt and V. Critchlow. Obesity and in creased growth following partial or complete isolation of ventromedial hypothalamus. Physiol. Behar. 7: 187-194, 1971.

36. Pickel, V. M., M. Segal and F. E. Bloom. A radioautography study of the efferent pathways of the nucleus locus coeruleus. $I$. comp. Neurol. 89: 312-348, 1974.

37. Raisman, G. The connections of the septum. Brain 89: $317-348$, 1966.

38. Raisman, G. An evaluation of the basic pattern of connections between the limbic system and the hypothalamus, $A m . J$. A $/ / a t$. 129: 197-202, 1970.

39. Rice, R. W., J. Kroning and V. Critchlow. Sex differences in the effects of surgical isolation of the medial basal hypothalamus on linear growth and plasma growth hormone levels of the rat. Endocrinolog: 98: 982-990, 1976.

40. Saller, C. F. and E. M. Stricker. Hyperphagia and increased growth in rats after intraventricular injection of 5,7 dihydroxytryptamine. Sciche 192: 385-387. 1976.

41. Sclafani, A. and S. P. Grossman. Hyperphagia produced by knife cuts between the medial and lateral hypothalamus in the rat. Phriol. Behai. 4: 533-537, 1969.

42. Shute, C. C. D. and P. R. Lewis. The ascending cholinergic reticular system: neocortical olfactory and subcortical projections. Brain 90: 497-540, 1967.

43. Slob, A. K. and J. J. Van der Werff ten Bosch. Sex differences in body growth in the rat. Physiol. Behas. 14: 353-361, 1975.

44. Storm-Mattisen, T. and H. C. Guldberg. 5-Hydroxytryptamine and noradrenaline in the hippocampal region: Effect of transection of afferent pathways on endogenous levels, high affinity uptake and some transmitter-related enzymes, $f$. Neurochem. 22: 793-803, 1974.

45. Swanson, L. W. and W. M. Cowan. An autoradiographic study of the organization of efferent connections of the hippocampal formation in the rat. J. comp. Neurol 172: 49-84, 1977.

46. Willoughby, J.O. and J. B. Martin. The suprachiasmatic nucleus synchronizes growth hormone secretory rhythms with the light-dark cycle. Brwin Res. 151: 413-417, 1978.

47. Wilson, M. and V. Critchlow. Effect of fornix transection or hippocampectomy on rhythmic pituitary-adrenal function in the rat. Neuroendocrinolog' 13: 29-40, 1973/1974

48. Wilson, M. and V. Critchlow. Effect of septal ablation on rhythmic pituitary-adrenal function in the rat. Nesurocendocrinology 14: 333-344, 1974. 